\title{
Door to groin time in patients with acute ischemic stroke presenting during on- vs off-hours, results from the MR CLEAN Registry
}

Wouter H Hinsenveld (MD) ${ }^{1}$, Inger R de Ridder (MD, PhD) ${ }^{1}$, Robert $\mathbf{J}$ van Oostenbrugge (MD, PhD) ${ }^{1}$, Jan A Vos (MD, PhD) ${ }^{2}$, Geert Lycklama a Nijeholt (MD, PhD) ${ }^{3}$, Jelis Boiten (MD, PhD) ${ }^{3}$, Wouter Schonewille (MD, PhD) ${ }^{2}$

1: Maastricht University Medical Center, Maastricht, The Netherlands; 2: Sint Antonius Hospital, Nieuwegein, The Netherlands; 3: Haaglanden Medical Center, The Hague, The Netherlands.

\section{Background and aim}

A large proportion of patients with acute ischemic stroke receiving endovascular treatment for large vessel occlusion (LVO) present during evenings, nights, weekends or holidays.

The aim of this study is to compare workflow times and functional outcome between patients presenting during on- and off-hours.

\section{Methods}

We analyzed data from the MR CLEAN Registry, a prospective, multicenter, observational study in the Netherlands that included patients with anterior circulation LVO treated with endovascular therapy (EVT) between March 2014 and June 2016.

Off-hours were defined as time of arrival at the intervention center (IC) or primary stroke center (PC) between 17:00 and 08:00, on weekends (Friday 17:00 to Monday 08:00) or Dutch National Holidays.

Workflow outcomes were corrected with linear regression for age, IVT, NIHSS, local anesthesia only and repeated imaging. Clinical and safety outcomes were corrected with logistic regression for age, IVT, NIHSS, collaterals, preMRS and onset to groin puncture time

\section{Results}

A total of 1488 patients were included for analysis. Of these, 935 patients $(62.8 \%)$ presented during off-hours. More patients were transferred from primary stroke centers (PC) to intervention centers (IC) during off-hours (48.3\% vs $57.9 \%$, p <0.001).

Door to groin time was significantly longer during off-hours. For direct referrals, the time interval after imaging was responsible for most of the delay. Transferred patients had most of their delay in the time interval before the IC.

Functional outcome was not significantly different between onand off-hour presentation. There was no difference in complication rates.

\begin{tabular}{|l|r|r|r|}
\hline Baseline variables & $\begin{array}{r}\text { On-hours } \\
\mathbf{N = 5 5 3}\end{array}$ & $\begin{array}{r}\text { Off-hours } \\
\mathbf{N = 9 3 5}\end{array}$ & P \\
\hline Age & $71.0(60.0-80.0)$ & $70.0(59.0-79.0)$ & 0.16 \\
\hline Men & $280(50.6 \%)$ & $514(55.0 \%)$ & 0.10 \\
\hline NIHSS & $15.0(11.0-20.0)$ & $16.0(12.0-20.0)$ & 0.70 \\
\hline Pre-mRS $\geq 3$ & $73(13.5 \%)$ & $98(10.7 \%)$ & 0.11 \\
\hline IVT performed & $439(79.7 \%)$ & $722(77.3 \%)$ & 0.29 \\
\hline Transfers & $267(48.3 \%)$ & $541(57.9 \%)$ & $<0.001$ \\
\hline LA only & $311(59.7 \%)$ & $499(59.3 \%)$ & 0.90 \\
\hline Last seen well & $138(25.0 \%)$ & $233(24.9 \%)$ & 0.99
\end{tabular}

Continuous and categorical variables are displayed using median and interquartile range. Pre-mRS: modified Rankin Scale at baseline. LA only: Local Anesthesia only, no general anesthesia or conscious sedation performed.

\begin{tabular}{|c|c|c|}
\hline Regression analysis & \multicolumn{2}{|c|}{ Delay in minutes $(95 \% \mathrm{Cl})$} \\
\hline First door to groin & & $14.76(9.09-20.43)$ \\
\hline Door to needle & & $3.39(0.48-6.29)$ \\
\hline Stratification & $\begin{array}{r}\text { Direct Referrals } \\
\text { Delay in minutes }(95 \% \mathrm{Cl})\end{array}$ & $\begin{array}{r}\text { Transfers } \\
\text { Delay in minutes }(95 \% \mathrm{Cl})\end{array}$ \\
\hline First door to groin* & $16.85(9.87-23.84)$ & $12.49(3.85-21.12)$ \\
\hline Door to needle & $2.84(-0.73-6.41)$ & $3.66(-0.74-8.07)$ \\
\hline PC door to IC door & $N / A$ & $9.19(1.86-16.51)$ \\
\hline IC door to groin & $N / A$ & $3.17(-1.95-8.29)$ \\
\hline
\end{tabular}

Displayed are beta coefficients adjusted for confounders using imputed data which indicate the delay between on-hour and off-hour presentation in minutes. PC: Primary stroke center; IC: Intervention Center. $: p=0.475$ for the interaction of transfer status on off-hour delay

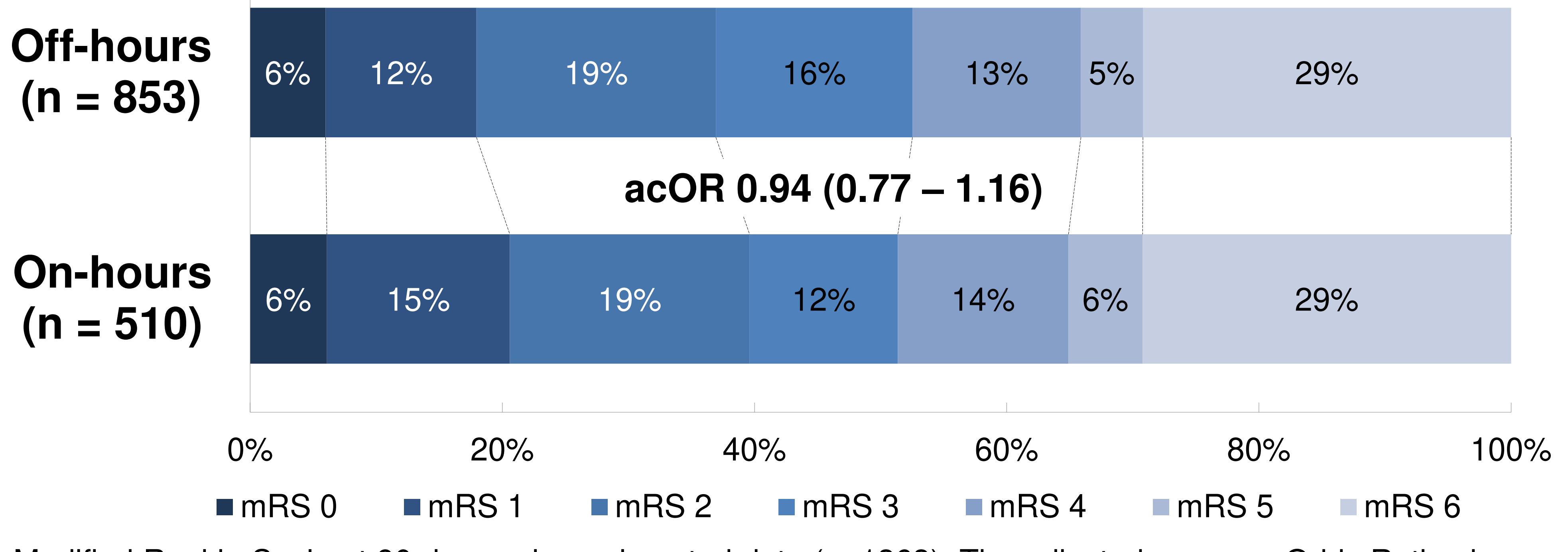

Modified Rankin Scale at 90 days using unimputed data $(n=1363)$. The adjusted common Odds Ratio shows the odds of a positive shift on the mRS scale of 1 point when patients present during off-hours.

\section{Conclusion}

Off-hour presentation for patients presenting with acute large vessel occlusion stroke seems to be associated with a door to groin delay of 15 minutes.

This delay may be more pronounced in direct referrals when compared to transferred patients. This delay doesn't seem to lead to worse functional outcome or higher complication rates. 